\title{
Early neurological care of patients with spinal cord injury
}

\author{
Thomas M. Kessler ${ }^{1} \cdot$ Luca R. Traini $^{2} \cdot$ Blayne Welk $^{3} \cdot$ Marc P. Schneider $^{1,4,5} \cdot$ Jeffrey Thavaseelan $^{6,7} \cdot$ Armin Curt $^{2}$
}

Received: 3 January 2018 / Accepted: 17 May 2018 / Published online: 28 May 2018

(c) Springer-Verlag GmbH Germany, part of Springer Nature 2018

\begin{abstract}
Purpose Considering the major clinical challenges of managing patients with spinal cord injury (SCI), we summarized the relevant aspects of the early (within 1 year after SCI) neurological care emphasizing common standards.

Methods This review was performed according to the methodology recommended by the Joint SIU-ICUD International Consultation. Embase and Medline databases were used to identify literature relevant to the early neurological care of SCI patients. Recommendations were developed by consensus and graded using a modified Oxford system which identifies level of evidence (LOE) and grade of recommendation (GOR).

Results Emergency health care providers must address altered mental status, evidence of intoxication, suspected extremity fracture/distracting injury, focal neurological deficit and spinal pain/tenderness to determine the risk of SCI in a trauma patient [LOE 1, GOR A]. Neurogenic shock must be recognized and treated [LOE 3, GOR A]. Spine surgeons should consider early decompression and spine fixation/stabilization, where indicated, and should promote early active rehabilitation to improve functional recovery [LOE 2, GOR B]. Clinicians should refer SCI patients to specialized SCI rehabilitation centers [LOE 4, GOR B], must apply interventions to prevent venous thromboembolism (i.e., compression devices and low-molecular weight heparin/unfractionated heparin) after acute SCI respecting contraindications [LOE 1, GOR A]. Contemporary guidelines to manage pain must be employed [LOE 1, GOR A] and methods to reduce the risk of pressure ulcers should be used [LOE 3, GOR B].

Conclusions Early treatment, prevention of associated complications and individualized patient-targeted rehabilitation programs provided by a specialized interdisciplinary team are crucial to optimize the outcome after SCI.
\end{abstract}

Keywords Spinal cord injury $\cdot$ Neurology $\cdot$ Early management

\section{Introduction}

Thomas M. Kessler

tkessler@gmx.ch

Luca R. Traini

lucartraini@gmail.com

Blayne Welk

bkwelk@gmail.com

Marc P. Schneider

mpschneider@outlook.com

Jeffrey Thavaseelan

jeff@perthurologyclinic.com.au

Armin Curt

armin.curt@balgrist.ch

1 Neuro-Urology, Spinal Cord Injury Center, Balgrist University Hospital, University of Zürich, Forchstrasse 340, 8008 Zürich, Switzerland
The care of patients with acute spinal cord injury (SCI) is rather complex and spans from the acute scene of injury, involves emergency rescue teams and transportation for

2 Neurology, Spinal Cord Injury Center, Balgrist University Hospital, University of Zürich, Zürich, Switzerland

3 St. Joseph's Health Care London and Western University, London, Canada

4 Brain Research Institute, University of Zürich, Zürich, Switzerland

5 Department of Health Sciences and Technology, Swiss Federal Institute of Technology, Zürich, Switzerland

6 St. John of God Murdoch Hospital, St. John of God Health Care, Perth, WA, Australia

7 University of Notre Dame, Perth, WA, Australia 
primary emergency hospital services and requires triage activities to initiate timely treatment in the intensive care unit and eventual early surgical interventions. Patients with SCI are cumbered with very vulnerable clinical conditions, and require comprehensive and coordinated care. This includes not only issues related to spine stability (including requirements for transportation and positioning), but also sensitive monitoring and treating cardiovascular impairments. Due to the complexity of an acute SCI (majority of patients suffer from a polytrauma), these patients should be immediately referred to primary acute trauma centers experienced to assess and manage any spine or spinal cord damage. For this purpose, multidisciplinary well-trained and coordinated teams are essential in applying appropriate treatments timely and handling medical challenges.

The objective of this paper is to outline common standards and supporting evidence for the neurological/medical care of patients within the first year after SCI.

\section{Methodology}

This review article was performed according to the methodology recommended by the Joint SIU-ICUD International Consultation (https://www.siu-urology.org/socie ty/siu-icud). We searched the Embase ${ }^{\circledR}$ and MEDLINE ${ }^{\circledR}$ databases from 1946 to November 2016 for studies and reviews on the early neurological care of patients with SCI and performed a search update in April 2018. No restrictions based on language or study type were applied. We additionally searched the reference lists of all included studies and any relevant review articles. Important studies were included and summarized in a narrative fashion. Recommendations were developed by consensus and graded using a modified Oxford system which identifies level of evidence (LOE) and grade of recommendation (GOR) (Table 1). The full version of this review with additional details and references has been published as chapter 2 in "Urologic Management of the Spinal Cord Injured Patient. A Joint SIU-ICUD International Consultation", Buenos Aires, Argentina, October 22, 2016: https://www.siu-urolo gy.org/society/siu-icud.

\section{Rescue and emergency care}

The principles of the Advanced Trauma Life Support ${ }^{\circledR}$ (ATLS ${ }^{\circledR}$ ) program by the American College of Surgeons advocate that a cervical spine injury should be assumed until proven otherwise in all trauma patients. The probability of a multilevel spinal injury in the setting of a known injury is approximately $20 \%$, necessitating complete spinal immobilization for any suspected SCI. The classic recommendations of the American College of Surgeons include a hard backboard, a rigid cervical collar, lateral support devices, and tape or straps to secure the patient, the collar and the lateral support devices to the backboard [1].

A rigid collar should be applied to the neck with manual in-line stabilization while minimizing cervical spine displacement. Spinal immobilization is a priority in the prehospital trauma algorithms and leads to improved outcomes [2]. While cervical collars and backboards are important tools in reducing further neurological injury, especially in blunt trauma, they have their own inherent risks and complications, such as high risk of aspiration, pressure sores and increased intracranial pressure. Practitioners must recognize these risks and understand the need to remove immobilizing

Table 1 Recommendations for the early neurological management of patients with SCI

\begin{tabular}{|c|c|c|}
\hline Recommendation & $\begin{array}{l}\text { Level of } \\
\text { evidence }\end{array}$ & $\begin{array}{l}\text { Grade of } \\
\text { recom- } \\
\text { mendation }\end{array}$ \\
\hline $\begin{array}{l}\text { Emergency health care providers must use the following criteria to determine the risk of SCI in a trauma patient: altered } \\
\text { mental status, evidence of intoxication, suspected extremity fracture/distracting injury, focal neurological deficit and } \\
\text { spinal pain/tenderness }\end{array}$ & 1 & A \\
\hline $\begin{array}{l}\text { Emergency health care providers must determine if airway and ventilator support are needed in patients with acute high } \\
\text { tetraplegia }\end{array}$ & 4 & A \\
\hline Emergency health care providers must recognize and treat neurogenic shock & 3 & A \\
\hline $\begin{array}{l}\text { Spine surgeons should consider early surgical spinal canal decompression and spine fixation/stabilization, where indi- } \\
\text { cated, and should promote early active rehabilitation to improve functional recovery }\end{array}$ & 2 & B \\
\hline Clinicians should refer patients with SCI to specialized SCI rehabilitation centers & 4 & B \\
\hline Clinicians must consider compression devices to prevent venous thromboembolism after SCI & 1 & A \\
\hline $\begin{array}{l}\text { Clinicians must begin low-molecular weight heparin/unfractionated heparin early after SCI, when there is no contrain- } \\
\text { dication }\end{array}$ & 1 & A \\
\hline Clinicians must employ contemporary guidelines to manage pain in SCI patients & 1 & A \\
\hline Clinicians should employ methods to reduce the risk of pressure ulcers in SCI patients & 3 & B \\
\hline
\end{tabular}


devices as soon as safety allows, particularly when concomitant head injury exists, in patients with ankylosing spondylitis, and in the setting of delayed resuscitation [3-5].

Prehospital spinal immobilization is required in the following circumstances:

- Spinal pain or tenderness, including any neck pain with a history of trauma

- Significant multiple system trauma

- Severe head or facial trauma

- Numbness or weakness in any extremity after trauma

- Loss of consciousness caused by trauma

- If mental status is altered (including drugs, alcohol, trauma) and no history is available, or the patient is found in a setting of possible trauma (e.g., lying at the bottom of the stairs or in the street), or the patient experienced near drowning with a history or probability of diving)

- Any significant distracting injury

Prehospital selective immobilization protocols (also known as clearance protocols) aim to identify trauma patients who are at very low risk of sustaining a SCI and, therefore, could be transported without spinal immobilization. Hoffman et al. [6] have validated five criteria-National Emergency X-Radiography Utilization Study (NEXUS) criteria-where patients' cervical spine can be cleared without imaging, these include:

1. No midline cervical tenderness

2. No altered mental status

3. No evidence of intoxication

4. No painful distracting injuries

5. No focal neurological deficits

Although there is no definite timeline for early transfer to surgery for isolated cervical SCI, it is recommended that patients reach spinal units within 8-24 h following injury [7]. An injured spinal cord is particularly susceptible to systemic hypoperfusion. Sound preclinical evidence and numerous retrospective clinical studies report that prompt hemodynamic resuscitation and blood pressure support improve neurological outcomes [8]. Hypotension should be corrected as soon as possible and mean arterial blood pressure should be maintained $>80 \mathrm{mmHg}$. Hypotension may be a result of neurogenic shock, but other potential causes of hemodynamic instability, such as hemorrhage, tension pneumothorax, cardiac tamponade and sepsis, should be considered and managed appropriately if present.

There is no international consensus for the application of high-dose methylprednisolone sodium succinate (MPSS) in acute traumatic SCI and recent recommendation considers MPSS rather as a treatment option than a standard of care $[9,10]$.
Recent evidence has emerged to suggest that surgery within $24 \mathrm{~h}$ of injury may improve neurological recovery and reduce adverse events [11]. Surgical approaches (anterior, posterior or combined) depend on the spinal conditions while in patients without spinal compression (such as hyperextension injury) posterior fixation and fusion will provide spinal stability. Anterior decompression and fusion with/ without posterior stabilization are required for patients with spinal canal compression. In some cases, anterior and posterior surgical approaches may be equivalent. Early (mostly considered to be less than $24 \mathrm{~h}$ ) surgical decompression is playing an increasing role in the treatment of acute SCI. Preliminary results of the Surgical Treatment for Acute Spinal Cord Injury Study (STASCIS) suggest decompression of the spinal cord within $24 \mathrm{~h}$ of injury is associated with improved neurological recovery in patients with cervical injury [12-16].

\section{Rehabilitation}

Inpatient SCI rehabilitation may last up to 6 months for tetraplegic and about 3 months for paraplegic patients and outpatient rehabilitation can extend beyond a year after injury. Primary rehabilitation should be performed in specialized SCI centers and adhere to international standards.

\section{Early stages of rehabilitation}

In the early stages after SCI, patients must be trained on proper positioning in bed and wheelchair either through independent movement or with the assistance of medical providers/caregivers to relieve pressure sensitive areas susceptible to pressure ulcers. Verticalization at the bedside is the first step in preparing the patient to sit in a wheelchair and often requires the help of two people. Physiotherapists and occupational therapists are involved in supporting and training for mobilization. For mobilization into an upright position (verticalization), especially in tetraplegia or in high paraplegia, it is important to gradually train the patient. Verticalization training should be at least 30 min every day and can be increased in a stepwise manner. If the patient is able to sit for at least $10 \mathrm{~min}$, a first transfer into the wheelchair can be performed [using a transfer board or a Hoyer ${ }^{\circledR}$ lift (Hoyer GmbH, Butzbach, Germany)]. After any mobilization, the skin should be inspected carefully for pressure ulcers when the patient is back in bed to allow any counter action be taken timely (i.e., modify the wheelchair or the seat (cushion) as well as the orthoses).

\section{Rehabilitation of impairment}

Controlled motor function (i.e., strength) is a prerequisite for training any activities of daily living (i.e., standing and 
walking) as well as self-care (feeding, bathing, dressing and grooming). Besides individual physiotherapy, starting with passive-assistive movements and slowly increasing with active movements (first without and then with resistance) for the upper and lower extremities, the usage of training devices (specific devices for medical training therapy) and weightlifting can help to improve motor strength. For any movement, appropriate preparation of muscle tone, assessment of joint conditions (i.e., assessing for signs of joint contractures), and allowing for meaningful sensory feedback are requirements in relearning and training limb movements. Crutches, canes and walkers can be integral tools in training for complex activities such as standing and walking.

\section{Rehabilitation of function}

When sitting in a wheelchair, it is important that the patient can rely on trunk stability to allow for a controlled and safe sitting position. Being able to transfer from the bed to the wheelchair and vice versa is an important milestone in preparing the patient for wheelchair training. Eventually, if a patient gained adequate sitting control and upper limb strength, wheelchair training may be started. The patient should learn to use the wheelchair without any support and eventually to overcome curbs and wheeling downhill independently. Finally, training moves to the real world conditions (outside of the hospital) and patients should reach independence in urban situations (e.g., tram, train, bus, etc.). Walking training becomes applicable in complete SCI patients with a lesion at least below the T11/12 level. Walking training for patients with incomplete SCI begins with provisional tools: driven gait orthoses [e.g., Lokomat ${ }^{\circledR}$ (Hocoma, Volketswil, Switzerland); Fig. 1], other various orthoses, treadmills, walkers and special shoes and footwear. Body weight-supported training (BWST) dynamically supports the patient's weight while attempting locomotion on a treadmill or over ground. With advances in technology, robot-assisted gait training (RAGT) with high intensity has become the standard of care after acute SCI. In addition, functional electrical stimulation (FES) for improving muscle function has been associated with improved functional outcomes.

\section{Rehabilitation of independence}

Rehabilitation of independence is an integral part of the rehabilitation program, as it defines the overall achieved level of outcome. While independence initially focuses on self-care issues (e.g., eating, dressing, toileting), it eventually covers areas of independent living, mobility, ranging from wheelchair use to independent and unsupervised walking.

\section{Management of non-genito-urinary problems}

\section{Respiration}

Respiratory complications represent the major cause of morbidity and mortality in SCI patients [17]. Prevention of respiratory complications must start immediately, irrespective of the level of the SCI. Secretion stagnation due to weakness of the expiratory musculature is treated with respiratory
Fig. 1 Driven gait orthoses (like the Lokomat ${ }^{\circledR}$ ) provide advanced technical means to enable and intensify locomotor training in patients with severe SCI where independent overground walking is not yet feasible

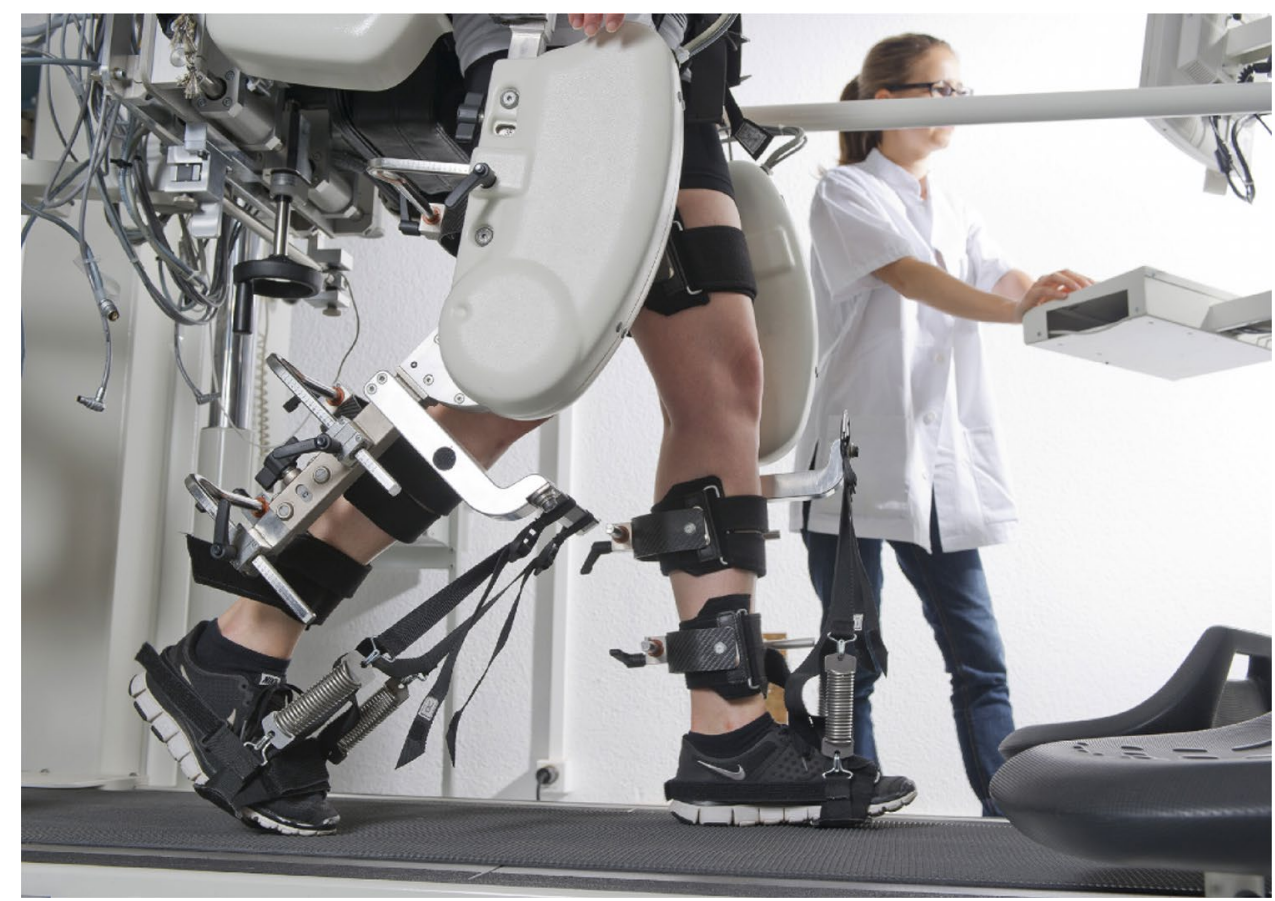


physiotherapy, postural drainage, tracheal suction, manual cough support and mechanical insufflation-exsufflation. Mechanical insufflation-exsufflation employs positive airway pressure (insufflation) using a mechanical device (cough assist machine) and then immediately afterwards transforms this positive pressure into negative pressure (exsufflation). The high expiration flow causes secretions to be forced up into the upper airways. The cough device can be applied via a face mask or even tracheostomy tube.

\section{Thromboembolism}

Pulmonary embolism is a potentially life-threatening condition and a significant cause of morbidity and mortality in patients with SCI, especially in cases of immobility. Prophylaxis is fundamental and includes compression devices to prevent venous thromboembolism in parallel with lowmolecular weight heparin (LMWH) early after SCI. Unfractionated heparin should be reserved for cases where LMWH is not available or contraindicated. If there are concerns of increased risk of bleeding, daily assessments should be performed for appropriate adjustment of LMWH [18].

\section{Spasticity}

Spasticity is a motor disorder characterized by a velocitydependent increase in tonic stretch reflexes (muscle tone) with exaggerated tendon jerks, resulting from hyperexcitability of the stretch reflex, as one component of the upper motor neuron syndrome [19]. Untreated, spasticity leads to contractures, which are often difficult to correct. Spasticity affects positioning and prevention of pressure ulcers. It makes hygiene tasks, especially cleaning of hands, axillae, elbows, and genital areas, particularly difficult. Spasticity can interfere with bowel and bladder care and sexual relationships.

Treatment of spasticity is individualized with the rational being that spasticity may not always have a negative impact on function. In some cases, it may provide benefits for standing and gait, as well as for transfers.

Stretching remains the essential principle for management of all patients with SCI and should form the base on which further treatments are added. Although splinting with a device such as an ankle-foot orthosis (AFO) is most commonly used to improve function by preventing excessive plantar flexion during the gait cycle (either passive due to weakness or active due to tone), splinting may also improve spasticity and certainly reduce the risk of contracture. Proper positioning in the bed and wheelchair can reduce the risk of contracture and may influence the degree of spasticity.

Physical modalities to treat spasticity include ultrasound, cryotherapy, vibration, shockwave therapy, magnetic stimulation, transcutaneous electrical nerve stimulation (TENS) and functional electrical stimulation (FES), and work by modulating the viscoelastic properties of muscles and tendons (cryotherapy, ultrasound, and shockwave therapy), inducing long-term depression at the spinal level, stimulating cortico-cortical inhibitory pathways (magnetic stimulation), inducing short-term plasticity in injured spinal motor systems, or activating proprioceptive inputs (TENS).

Baclofen is the most widely used oral antispastic drug (starting dose $5 \mathrm{mg}$ thrice daily, increased by $5-10 \mathrm{mg}$ weekly until optimal effect is achieved with a maximum dose of $80-120 \mathrm{mg} /$ day (maximum dose depends on local jurisdictional regulations and exceeding the recommended maximum dose needs prior discussion with the patient). Diazepam has somewhat fallen out of favor, given the adverse effects and the very long half-life time, particularly in the elderly. Clonazepam is a medication similar to diazepam, but has a shorter half-life time and, therefore, the effect will wear off more quickly when it is discontinued. It is particularly useful in the treatment of nocturnal spasms that interfere with sleep. Tizanidine and clonidine act centrally at both supraspinal and spinal sites. Dantrolene with its direct effect on the muscle has as major drawback with a potential for serious hepatotoxicity. Gabapentin may be a reasonable medication in situations in which both neuropathic pain and spasticity are limiting, or in which it is suspected that the neuropathic pain is the primary noxious stimulus leading to increased spasticity (starting dose $300 \mathrm{mg}$ once daily on day $1,300 \mathrm{mg}$ twice daily on day $2,300 \mathrm{mg}$ thrice daily on day 3 , then increased according to the patient's response in steps of $300 \mathrm{mg}$ every 2 to 3 days to maximum of 3'600 mg daily).

Local treatment with focal neurolysis or chemodenervation has been used as a spasticity management tool in a variety of upper motor neuron disorders (in the past phenol solutions, currently botulinum toxin A or B).

Intrathecal baclofen is indicated in patients with severe spasticity who are unresponsive to oral baclofen and have failed other conservative and pharmacologic therapies, and/ or who experience unacceptable side effects at effective doses of oral medications.

Surgical procedures (such as laminectomy, cordectomy, adhesiolysis of spinal cord tethering and syringomyelia, tendon lengthening, tendon transfer, and osteotomy) may be considered in the case that patients do not respond to typical physical, pharmacologic and intrathecal options, although it is quite uncommon in patients with SCI.

\section{Bowel dysfunction}

Bowel dysfunction is a major physical and psychological burden for patients with SCI [20]. Spinal cord lesions affect colorectal motility, anorectal sensation and anal sphincter function. They can also cause neurogenic constipation. As 
with striated muscles, there are two distinct patterns in the clinical presentation of bowel dysfunction: injury above the conus medullaris and injury at the conus medullaris and/or cauda equina [21]. Bowel dysfunction caused by a lesion above the conus medullaris (hyperreflexic bowel) is characterized by increased colonic wall and anal tone. Voluntary (cortical) control of the external anal sphincter is disrupted, and the sphincter remains tight, thereby promoting retention of stool. The nerve connections between the spinal cord and the colon remain intact, and therefore, there is preserved reflex coordination and stool propulsion. The typical clinical presentation of bowel dysfunction is constipation and fecal retention, due, at least in part, to external anal sphincter activity. In these individuals, stool regulation occurs by reflex activity, caused by a stimulus introduced into the rectum, such as an irritant suppository or digital stimulation. Lesions of the conus medullaris/cauda equina are associated with an immediate damage of efferent motor pathways innervating the pelvic muscles with a predominant pattern of atonic (i.e., flaccid) anal sphincter resulting in fecal incontinence.

An effective bowel management program for a person with a neurogenic bowel involves the modulation of stool consistency, promotion of stool transit through the bowel, and effective reflex or mechanical evacuation of stool from the rectum at an appropriate time and place. Such care is pre-emptive; bowel function is manipulated so that effective evacuation from the rectum occurs at a pre-specified and predictable time, when appropriate resources, such as caregiver assistance, are available. By emptying the bowel at a chosen time, incontinence is avoided. In addition, regular emptying reduces the risk of impaction of stool due to constipation [22].

\section{Pain}

Chronic pain is common in patients with SCI and the approach is similar to all chronic pain conditions: history, physical examination, and judicious use of diagnostic testing. Pain assessment should also include a patient self-report component. Regarding diagnostic testing, above-level syndromes can be evaluated in a manner parallel to a non-SCI patient. Conditions associated with at-level and below-level lesions are more challenging. Imaging of the site of the initial spinal injury should be considered in these circumstances. Potential examples of pain generators that might be detected include segmental instability or compression at the site of injury, spinal nerve impingement, orthopedic hardware loosening, fluid collection, and syringomyelia.

The treatment of central neuropathic pain is very challenging. As a rule, complete pain resolution is unlikely.

Non-pharmacologic management with a generalized exercise program in the form of global strength training, cardiovascular training or recreational physical activities has the potential to be beneficial in several SCI-related conditions (e.g., spasticity, muscle atrophy, and bone health), but its effect on global pain in these conditions has not been greatly satisfactory. In addition to generalized and specified exercise programs, referral to physical or occupational therapy may be appropriate for patients with SCI with musculoskeletal pain, in an effort to address the biomechanical abnormalities that can be associated with mobility aids. Modification of orthotics, canes, walkers, crutches and wheelchairs has the potential to influence detrimental ergonomics.

Pregabalin is the first choice for pharmacologic treatment of neuropathic pain (dosing from 150 to $600 \mathrm{mg} / \mathrm{day}$, usually in two divided doses) and also gabapentin is commonly used (starting dose $300 \mathrm{mg} /$ day, increased by $300-\mathrm{mg}$ increments every 4-7 days, initially to three times daily to a goal of 1'800 mg/day).

Antidepressants for below-level neuropathic SCI pain have a long-standing tradition, especially amitriptyline (initial dosage $10-25 \mathrm{mg}$ at bedtime with increases every 4-7 days to a goal of $75-100 \mathrm{mg}$ at bedtime) and more recently dual serotonin and norepinephrine reuptake inhibitors.

Opioid medications are applied but concerns over the potential exacerbation of neurogenic bowel because of opioid-related constipation makes the situation challenging. Nevertheless, there are new strategies for the management of opioid-related constipation, including peripheral opioid receptor antagonists and prokinetic agents [23].

Acupuncture and multiple surgical interventions [commissurotomy (i.e., midline myelotomy transecting the crossing fibers in the spinal cord), dorsal root entry zone lesioning, cordotomy (i.e., selective lesioning of the lateral spinothalamic tract pain pathways in the anterolateral cord contralateral to the side of pain) and cordectomy (i.e., transection of the spinal cord)] [24] attempting to interrupt aberrant ascending nociceptive signaling or stimulation procedures attempting to modulate this signaling (neuromodulation) have been proposed to treat very selected patients with refractory central neuropathic pain.

\section{Autonomic dysreflexia (AD)}

$\mathrm{AD}$ is a sudden and exaggerated autonomic response to various stimuli in patients with SCI or spinal dysfunction. It generally manifests in patients with SCI at or above T6, it is more common in complete versus incomplete SCI, it mostly presents for the first time within a year after SCI and it is defined by an increase in systolic blood pressure $>20 \mathrm{mmHg}$ from baseline [25, 26]. Furthermore, AD can have lifethreatening consequences if not properly managed [27]. $\mathrm{AD}$ is caused by spinal reflex mechanisms that are initiated 
when a noxious stimulus enters the spinal cord below the level of injury. This afferent stimulus generates sympathetic overactivity leading to vasoconstriction below the neurological lesion, along with involvement of splanchnic circulation causing vasoconstriction and hypertension. The excessive compensatory parasympathetic activity (and lack of sympathetic tone) leads to vasodilation above the level of the lesion and is thought to be responsible for headache, flushing, sweating, and nasal congestion. The reflex bradycardia is secondary to vagal stimulation. Bladder distension is the most common triggering factor for $\mathrm{AD}$. The distension that can result from urinary retention or catheter blockage accounts for up to $85 \%$ of cases [28]. The second most common triggering factor for $\mathrm{AD}$ is bowel distension due to fecal impaction. Other potential factors include hemorrhoids and anal fissures, gastrointestinal precipitants (appendicitis, cholecystitis, etc.), pressure ulcers, ingrown toenails, fractures, heterotopic ossification, menstruation, pregnancy or labor, deep vein thrombosis, pulmonary embolism and sexual activity. Medications, especially nasal decongestants and misoprostol, may also induce AD. Education of patients, caregivers, and family members regarding $\mathrm{AD}$ is vital to prevent it and to recognize its occurrence without delay.

If $\mathrm{AD}$ occurs, it is essential to find and eliminate the triggering stimulus (e.g., bladder distension or bowel impaction). Initial management also involves placing the patient in an upright position to take advantage of any orthostatic reduction in blood pressure, and loosening tight clothing and/or constrictive devices. Blood pressure should be monitored until the patient is stable. These steps will resolve the problem in most patients, but in some, pharmacotherapy (in general, antihypertensive agents that have a rapid onset and short duration of action) may become necessary.

\section{Pressure ulcers}

Pressure ulcers are a constant and costly problem in patients with SCI, typically resulting from partial or complete lack of control and sensation in sitting/lying contact areas, as well as from varying degrees of incontinence. Extended periods of immobility result in excessive tissue pressure and, ultimately, ulcerations. Up to $95 \%$ of individuals with SCI will develop pressure ulcers in their lifetime [29]. The most commonly used staging system for pressure ulcers was proposed in 1989 and updated in 2007 by the National Pressure Ulcer Advisory Panel (NPUAP) using a consensus conference model [30].

- Stage I: Intact skin with non-blanchable redness of a localized area usually over a bony prominence. Darkly pigmented skin may not have visible blanching; its color may differ from the surrounding area.
- Stage II: Partial thickness loss of dermis presenting as a shallow open ulcer with a red pink wound bed, without slough. May also present as an intact or open/ruptured serum-filled blister.

- Stage III: Full thickness tissue loss. Subcutaneous fat may be visible but bone, tendon or muscle is not exposed. Slough may be present but does not obscure the depth of the tissue loss. May include undermining and tunneling.

- Stage IV: Full thickness tissue loss with exposed bone, tendon or muscle. Slough or eschar may be present on some parts of the wound bed. Often include undermining and tunneling.

- Unstageable: Full thickness tissue loss in which the base of the ulcer is covered by slough (yellow, tan, gray, green or brown) and/or eschar (tan, brown or black) in the wound bed.

Pressure relief is the standard conservative treatment for a non-healing pressure ulcer and has to be patient-tailored.

Dressing of pressure ulcers can be characterized as passive action or active action, depending on the wound. Negativepressure wound therapy (NPWT) has become an important tool in the management of a wide spectrum of wounds. The vacuum-regulation device provides continuous or intermittent, controlled negative pressure to the wound through an airtight dressing, which is changed every second or third day.

Surgical management of pressure ulcers involves a spectrum of options: simple debridement with direct closure, skin grafting, fasciocutaneous flaps, myocutaneous flaps, combination proximal femoral osteotomy and flap reconstruction (Girdlestone procedure) or end-stage lower extremity disarticulation and total thigh flap.

Importantly, vacuum-regulation device and surgical correction of wounds are only instituted for more advanced pressure ulcers, particularly non-infected stage IV wounds.

\section{Conclusions}

Early treatment, prevention of associated complications and individualized patient-targeted rehabilitation programs provided by a specialized interdisciplinary team are crucial to optimize the outcome after SCI.

Recommendations for the early neurological management of patients with SCI are summarized in Table 1.

Author contributions Project development: TMK. Literature review and data extract: All. Manuscript writing: TMK, LT. Manuscript editing: BW, MPS, JT, AC. Consensus process for recommendations: All.

\section{Compliance with ethical standards}

Conflict of interest The authors declare no conflict of interest. 
Ethical approval As this is a review of the literature, no ethical approval was necessary.

\section{References}

1. Alexander RHPH (1993) Advanced trauma life support program for physicians: ATLS. American College of Surgeons, Chicago

2. Burton JH, Dunn MG, Harmon NR, Hermanson TA, Bradshaw JR (2006) A statewide, prehospital emergency medical service selective patient spine immobilization protocol. J Trauma 61(1):161-167

3. McHugh TP, Taylor JP (1998) Unnecessary out-of-hospital use of full spinal immobilization. Acad Emerg Med 5(3):278-280

4. Kolb JC, Summers RL, Galli RL (1999) Cervical collar-induced changes in intracranial pressure. Am J Emerg Med 17(2):135-137

5. Abram S, Bulstrode C (2010) Routine spinal immobilization in trauma patients: what are the advantages and disadvantages? Surgeon 8(4):218-222

6. Hoffman JR, Mower WR, Wolfson AB, Todd KH, Zucker MI (2000) Validity of a set of clinical criteria to rule out injury to the cervical spine in patients with blunt trauma. National Emergency X-Radiography Utilization Study Group. N Engl J Med 343(2):94-99

7. Furlan JC, Noonan V, Cadotte DW, Fehlings MG (2011) Timing of decompressive surgery of spinal cord after traumatic spinal cord injury: an evidence-based examination of pre-clinical and clinical studies. J Neurotrauma 28(8):1371-1399

8. Vale FL, Burns J, Jackson AB, Hadley MN (1997) Combined medical and surgical treatment after acute spinal cord injury: results of a prospective pilot study to assess the merits of aggressive medical resuscitation and blood pressure management. J Neurosurg 87(2):239-246

9. Hurlbert RJ, Hamilton MG (2008) Methylprednisolone for acute spinal cord injury: 5-year practice reversal. Can J Neurol Sci 35(1):41-45

10. Fehlings MG, Wilson JR, Tetreault LA, Aarabi B, Anderson P, Arnold PM, Brodke DS, Burns AS, Chiba K, Dettori JR, Furlan JC, Hawryluk G, Holly LT, Howley S, Jeji T, Kalsi-Ryan S, Kotter M, Kurpad S, Kwon BK, Marino RJ, Martin AR, Massicotte E, Merli G, Middleton JW, Nakashima H, Nagoshi N, Palmieri K, Skelly AC, Singh A, Tsai EC, Vaccaro A, Yee A, Harrop JS (2017) A clinical practice guideline for the management of patients with acute spinal cord injury: recommendations on the use of methylprednisolone sodium succinate. Glob Spine J 7(3 suppl):203S-211S

11. Wilson JR, Arnold PM, Singh A, Kalsi-Ryan S, Fehlings MG (2012) Clinical prediction model for acute inpatient complications after traumatic cervical spinal cord injury: a subanalysis from the Surgical Timing in Acute Spinal Cord Injury Study. J Neurosurg Spine 17(1 Suppl):46-51

12. Albin MS, White RJ (1987) Epidemiology, physiopathology, and experimental therapeutics of acute spinal cord injury. Crit Care Clin 3(3):441-452

13. Campagnolo DI, Esquieres RE, Kopacz KJ (1997) Effect of timing of stabilization on length of stay and medical complications following spinal cord injury. J Spinal Cord Med 20(3):331-334

14. Carlson GD, Gorden CD, Oliff HS, Pillai JJ, LaManna JC (2003) Sustained spinal cord compression: part I: time-dependent effect on long-term pathophysiology. J Bone Joint Surg Am 85-A(1):86-94

15. Cengiz SL, Kalkan E, Bayir A, Ilik K, Basefer A (2008) Timing of thoracolomber spine stabilization in trauma patients; impact on neurological outcome and clinical course. A real prospective (rct) randomized controlled study. Arch Orthop Trauma Surg 128(9):959-966

16. Fehlings MG, Vaccaro A, Wilson JR, Singh ADWC, Harrop JS, Aarabi B, Shaffrey C, Dvorak M, Fisher C, Arnold P, Massicotte EM, Lewis S, Rampersaud R (2012) Early versus delayed decompression for traumatic cervical spinal cord injury: results of the surgical timing in acute spinal cord injury study (STASCIS). PLoS One 7(2):e32037

17. DeVivo MJ, Krause JS, Lammertse DP (1999) Recent trends in mortality and causes of death among persons with spinal cord injury. Arch Phys Med Rehabil 80(11):1411-1419

18. Paralyzed Veterans of America (PVA): clinical practice guidelines: prevention of venous thromboembolism in individuals with spinal cord injury. https://www.pva.org/CMSPages/ GetFile. aspx? guid=9243b370-f0d3-47da-8b7f-44f86182a6c8. Accessed 9 Apr 2018

19. Lance J (1980) Pathophysiology of spasticity and clinical experience with baclofen. Spasticity: disordered motor control. Yearbook, Chicago

20. Cotterill N, Madersbacher H, Wyndaele JJ, Apostolidis A, Drake MJ, Gajewski J, Heesakkers J, Panicker J, Radziszewski P, Sakakibara R, Sievert KD, Hamid R, Kessler TM, Emmanuel A (2017) Neurogenic bowel dysfunction: clinical management recommendations of the Neurologic Incontinence Committee of the Fifth International Consultation on Incontinence 2013. Neurourol Urodyn. https://doi.org/10.1002/nau.23289

21. Singal AK, Rosman AS, Bauman WA, Korsten MA (2006) Recent concepts in the management of bowel problems after spinal cord injury. Adv Med Sci 51:15-22

22. Coggrave MAD, Adcock C, Brown A, Davies D, Dehal-Clark A, et al (2012) Guidelines for management of neurogenic bowel dysfunction in individuals with central neurological conditions. Multidisciplinary Association of Spinal Cord Injured Professionals (MASCIP)

23. Walters JB, Montagnini M (2010) Current concepts in the management of opioid-induced constipation. J Opioid Manag $6(6): 435-444$

24. Kanpolat Y (2004) The surgical treatment of chronic pain: destructive therapies in the spinal cord. Neurosurg Clin N Am 15(3):307-317

25. Krassioukov A, Biering-Sorensen F, Donovan W, Kennelly M, Kirshblum S, Krogh K, Alexander MS, Vogel L, Wecht J (2012) International standards to document remaining autonomic function after spinal cord injury. J Spinal Cord Med 35(4):201-210

26. Panicker JN, Fowler CJ, Kessler TM (2015) Lower urinary tract dysfunction in the neurological patient: clinical assessment and management. Lancet Neurol 14(7):720-732

27. Liu N, Zhou M, Biering-Sorensen F, Krassioukov AV (2015) Iatrogenic urological triggers of autonomic dysreflexia: a systematic review. Spinal Cord 53(7):500-509

28. Shergill IS, Arya M, Hamid R, Khastgir J, Patel HR, Shah PJ (2004) The importance of autonomic dysreflexia to the urologist. BJU Int 93(7):923-926

29. Garber SL, Rintala DH, Hart KA, Fuhrer MJ (2000) Pressure ulcer risk in spinal cord injury: predictors of ulcer status over 3 years. Arch Phys Med Rehabil 81(4):465-471

30. Black J, Baharestani M, Cuddigan J, Dorner B, Edsberg L, Langemo D, Posthauer ME, Ratliff C, Taler G (2007) National Pressure Ulcer Advisory Panel's updated pressure ulcer staging system. Dermatol Nurs 19(4):343-349 